\title{
Multitask machine learning models for predicting lipophilicity (logP) in the SAMPL7 challenge
}

\author{
Eelke B. Lenselink ${ }^{1}$ (D) . Pieter F. W. Stouten ${ }^{1}$ (D)
}

Received: 24 February 2021 / Accepted: 22 June 2021 / Published online: 17 July 2021

(c) The Author(s) 2021

\begin{abstract}
Accurate prediction of lipophilicity- $\log \mathrm{P}$ - based on molecular structures is a well-established field. Predictions of $\log \mathrm{P}$ are often used to drive forward drug discovery projects. Driven by the SAMPL7 challenge, in this manuscript we describe the steps that were taken to construct a novel machine learning model that can predict and generalize well. This model is based on the recently described Directed-Message Passing Neural Networks (D-MPNNs). Further enhancements included: both the inclusion of additional datasets from ChEMBL (RMSE improvement of 0.03), and the addition of helper tasks (RMSE improvement of 0.04). To the best of our knowledge, the concept of adding predictions from other models (Simulations Plus $\log \mathrm{P}$ and $\log \mathrm{D} @ \mathrm{pH} 7.4$, respectively) as helper tasks is novel and could be applied in a broader context. The final model that we constructed and used to participate in the challenge ranked 2/17 ranked submissions with an RMSE of 0.66, and an MAE of 0.48 (submission: Chemprop). On other datasets the model also works well, especially retrospectively applied to the SAMPL6 challenge where it would have ranked number one out of all submissions (RMSE of 0.35). Despite the fact that our model works well, we conclude with suggestions that are expected to improve the model even further.
\end{abstract}

Keywords D-MPNN $\cdot$ Multitask machine learning $\cdot \log P$ prediction $\cdot$ SAMPL7

\section{Introduction}

Lipophilicity plays a key role in drug discovery, from an indirect role in ADME (absorption, distribution, metabolism, and excretion), to toxicity and potency [1,2]. Typically, in drug discovery projects, lipophilicity predictions are used by the chemist to drive the chemistry forward, i.e. to balance lipophilicity and potency [2]. Often however, the predicted values of lipophilicity, or derivatives thereof (such as LLE) [3], are used as is, without considering the uncertainty or error in these predictions. Moreover, the performance of machine learning-based methods reported in the literature is typically an overestimation of the true performance of these models $[4,5]$, because historically they were based on a division into random training and test sets that were structurally not very different. Time- and scaffoldbased splits would provide more realistic assessments of the expected performance $[6,7]$.

Eelke B. Lenselink

bart.lenselink@glpg.com

1 Galapagos NV, Generaal De Wittelaan L11 A3, 2800 Mechelen, Belgium
Nevertheless, there are many well-performing programs/ models to estimate $\log \mathrm{P}$, and the reader is referred to Mannhold et al. [8] for a more detailed overview. Machine learning (ML) approaches that predict $\log \mathrm{P}$ come in two flavors: additive and property-based. Additive models such as $\mathrm{X} \log \mathrm{P} 3$ [9] assume additivity of $\log \mathrm{P}$ for different atom (types), while property-based models such as Simulations Plus (hereafter referred to as $\mathrm{S}+$ ) $\log \mathrm{P}[10]$ use statistical methods and molecular descriptors. A third flavor of methods, exemplified by COSMOtherm [11] is physics-based rather than the result of an ML approach. It is also widely used although the calculation speed is usually about an order of magnitude lower than for ML models.

The SAMPL blind prediction challenges have been a way to quantitively benchmark different methods. For example, the recent SAMPL6 challenge for $\log P$ assessed 91 different prediction methods [12]. Motivated by this opportunity to prospectively assess our approach to constructing multitask ML models, we decided to participate in the SAMPL7 challenge [13].

Recent work in ML has revealed the advantages of neural networks, especially when employing techniques such as: (1) learned representations, and (2) multitasking. 
1. Graph-based convolutional neural networks (GCNNs) have shown to hold promise (given sufficient data), ever since their application was first described [14]. For instance, weave, one type of GCNN, has been shown to prospectively outperform Random Forests (RFs) when applied to DNA-Encoded Library (DEL) screening data [15]. Recently, Directed-message passing neural networks (D-MPNNs) that are based on learned representations rather than fixed molecular descriptors have been introduced [6]. These D-MPNNs have shown to perform well across the board without (much) hyperparameter optimization. D-MPNNs iteratively generate representations of molecules by transmitting information across bonds (directed), in the message passing phase. Subsequently, in the readout phase, these representations are used to predict the property of interest. For further information refer to Yang et al. and $\mathrm{Wu}$ et al. $[6,16]$. D-MPNNs have shown to outperform RFs and other GCNNs across the board [6]. Moreover, in practice, D-MPNNs have been applied successfully to the discovery of novel antibiotics [17].

2. Another advantage of neural networks is their ability to learn multiple tasks simultaneously. The concept of developing one model for multiple tasks has been first utilized in the Kaggle bioactivity challenge hosted by Merck, where the winning team used a mix of singletask and multitask neural networks [18]. Subsequently, the added value has been demonstrated with several different datasets [19], such as for modelling of ChEMBL bioactivity data [20], and for ADME modelling [21, 22]. Overall, multitask models are particularly beneficial if the tasks are related [18].

In this manuscript the steps that were undertaken that led to our final model are illustrated. Starting from a test set that is similar to the molecules of the SAMPL7 set, the following steps were performed, and their effects evaluated:

1. Using a default D-MPNN architecture, and adding rdkit descriptors

2. Adding extra datasets as separate tasks: ChEMBL/AstraZeneca deposited dataset

3. Running a hyperparameter optimization

4. Adding $\log \mathrm{P} / \log \mathrm{D} 7.4(=\log \mathrm{D} @ \mathrm{pH} 7.4)$ predictions (calculated with S+ ADMET Predictor V9.5 [10]), either as descriptors or as tasks.

Overall, those steps led to the final model that scored second (out of 17 ranked submissions) in the SAMPL 7 LogP challenge.

\section{Methods}

\section{Datasets and test set creation}

Biovia's Pipeline Pilot v17.2.0.1361 [23] was used for most of the data processing steps.

The first set of $\log \mathrm{P}$ data was extracted from the Opera datasets [24], which contained 13,963 structure- $\log P$ datapoints.

Because we wanted to build a test set that mimicked the SAMPL7 challenge molecules, a tailored training/test set was created, as follows:

233 molecules were selected for the test set, based on their maximum similarity to the 22 SAMPL7 molecules [25] being greater than 0.25 (Tanimoto Coefficient (TC), ECFP_6 fingerprints [26], as implemented in Pipeline Pilot). To make the training/test split simultaneously more realistic and more challenging), the training set was constructed by taking the remainder of the molecules and filtering out 756 molecules with a similarity $>0.4$ compared to the test set (TC, ECFP_6 fingerprints) and one molecule with an incorrect smiles code, leading to a training set of 12,973 molecules.

5539 additional datapoints were extracted from ChEMBL_25 [27], using $\log \mathrm{P}$ as a query in the assay description. Calculated $\log \mathrm{P}$ datapoints were discarded. For the $\log$ D7.4 data we used all data available in the AstraZeneca deposited set (DOC ID: CHEMBL3301361).

Finally for models 9,10 , and $12, \mathrm{~S}+\log \mathrm{P}$ and $\log \mathrm{D} 7.4$ were calculated for all molecules using ADMET Predictor V9.5 [10]. For model 10 we added $S+\log \mathrm{P}$ and $\log \mathrm{D} 7.4$ as descriptors (in a separate input layer), while for model 9 those calculated properties were used as (helper) tasks. The model learns $\mathrm{S}+\log \mathrm{P}$ and $\log \mathrm{D} 7.4$ as additional tasks in the loss function on the basis of the structures. The use of these calculated properties as additional helper tasks could help regularize the model.

\section{D-MPNN training}

Directed message passing neural networks [6] were trained on a workstation containing a NVIDIA RTX6000. Rdkit [28] in python was used to convert the molecule files (sdf) into a format compatible for use in chemprop (with columns for smiles, $\log$ P, $\log$ P_Chembl, $\log$ D7.4_AZ, etc.).

Because an external test set was used, the test set was omitted in the training loop of chemprop (-split_sizes 0.9 0.10 ). A hyperparameter optimization run was performed using the hyperparameter_optimization.py script provided by chemprop, which uses hyperopt [29] to tune the hyperparameters of the neural network. The search grid was 
not changed, and a scaffold split (-split_type scaffold_balanced) was used for this evaluation. The settings that were used for the "optimized model" were: 5 message passing steps (-depth 5 ), dropout of 0 percent (-dropout 0 ), 3 feed forward layers (-ffn_num_layers 3 ), and 700 neurons in the hidden layers (-hidden_size 700).

Confidence intervals on the performance metrics were calculated using sklearn [30] and bootstrapping, using mlxtend [31].

After having developed the series of models from 1 through 12 in order to optimize the settings, we built the best possible model (12_Full) by creating an emsemble of 10 individual models on the basis of all available data (Opera, ChEMBL and AZ), without using a separate test set. Predictions submitted to the challenge were done on the basis of this Model 12_Full, while the Standard Error in the Mean (SEM) of the prediction for each compound was estimated on the basis of the 10 individual model predictions. For benchmarking purposes, a singletask ensemble model consisting of 10 individual models (11_Full) was developed using all Opera data.

\section{Other software}

The following additional tools were used throughout the work described in this manuscript: AlogP (through Biovia's Pipeline Pilot) [32] and XlogP3 [9].

\section{Results}

\section{Baseline performance}

The starting point for the experiments was the tailored, SAMPL7-biased dataset (see methods). For lipophilicity data we resorted primarily to the Opera dataset [24], which was used in one of the best performing models for the SAMPL6 challenge [12].

In our first set of experiments (Table 1: Models 1-3) we studied the impact of varying the settings and adding extra descriptors natively available in chemprop. "Out of the box" (default) the D-MPNN model already provided comparable performance to commercial solutions (RMSE model $1=0.45 ;$ RMSE $S+\log P=0.40)$. Having no knowledge of and no control over the training/test sets used to develop the commercial $\log \mathrm{P}$ predictors, the comparison was primarily done to establish a baseline for further experiments.

Adding extra rdkit descriptors (calculated by descriptastorus, a library included in the chemprop package) as a separate layer, (model 2) didn't improve the performance on this test set, and came at an extra computational cost. To analyze the relative contributions of the learned representation and the molecular descriptors, respectively, we also trained a network only based on rdkit descriptors (model 3). As expected, this model performed substantially worse (RMSE model $3=0.60$; RMSE model $1=0.45$ ).
Table 1 Overview of the optimization done on the model, performance $\left(\mathrm{R}^{2}\right.$, RMSE, Spearman $\rho$ ) on the test set constructed for this challenge

\begin{tabular}{lllll}
\hline Model & Description & $\mathrm{R}^{2}$ & $\mathrm{RMSE}$ & Spearman $\rho$ \\
\hline- & AlogP & $0.83[0.71,0.90]$ & $0.73[0.55,0.93]$ & $0.90[0.85,0.94]$ \\
- & XlogP3 & $0.85[0.75,0.92]$ & $0.67[0.48,0.87]$ & $0.91[0.87,0.95]$ \\
- & S+ $\operatorname{logP}$ & $0.95[0.91,0.97]$ & $0.40[0.32,0.48]$ & $0.97[0.94,0.98]$ \\
1 & default & $0.93[0.89,0.96]$ & $0.45[0.36,0.57]$ & $0.96[0.94,0.97]$ \\
2 & 1+rdkit & $0.93[0.89,0.96]$ & $0.45[0.37,0.55]$ & $0.96[0.94,0.98]$ \\
3 & rdkit only & $0.88[0.82,0.92]$ & $0.60[0.50,0.70]$ & $0.94[0.91,0.96]$ \\
4 & 1+ChEMBL merged & $0.88[0.81,0.92]$ & $0.60[0.51,0.71]$ & $0.94[0.92,0.96]$ \\
5 & 1+ChEMBL separate & $0.93[0.88,0.95]$ & $0.47[0.38,0.58]$ & $0.96[0.94,0.98]$ \\
6 & 5+AZ_logD7.4 & $0.94[0.91,0.96]$ & $0.42[0.35,0.50]$ & $0.97[0.95,0.97]$ \\
7 & 5+AZ_ADME & $0.94[0.90,0.96]$ & $0.44[0.36,0.51]$ & $0.97[0.95,0.98]$ \\
8 & 6+hyperopt parameters & $0.93[0.88,0.95]$ & $0.47[0.39,0.58]$ & $0.96[0.94,0.97]$ \\
9 & 6+S+logP/logD7.4 as tasks & $0.95[0.93,0.97]$ & $0.38[0.32,0.44]$ & $0.97[0.96,0.98]$ \\
10 & 6+S+logP/logD7.4 as descriptors & $0.95[0.92,0.97]$ & $0.39[0.34,0.44]$ & $0.97[0.96,0.98]$ \\
11 & 1, ensemble of 10 & $0.94[0.89,0.96]$ & $0.44[0.35,0.55]$ & $0.96[0.94,0.98]$ \\
12 & 9, ensemble of 10 & $0.95[0.92,0.97]$ & $0.39[0.33,0.46]$ & $0.97[0.96,0.98]$ \\
\hline
\end{tabular}

The ordinal model numbers in the left-most column indicate the sequence in which the models were developed: for example model $6\left(5+\mathrm{AZ} \_\operatorname{logD} 7.4\right)$ means that the settings/data of model 5 were used and the AZ_logD7.4 data were added. The $95 \%$ confidence interval for the different performance metrics is shown between square brackets 


\section{Adding datasets as separate endpoints}

To further improve the model performance and generalizability, two data sets were added to the Opera set (see methods):

1. Experimental $\log P$ data from ChEMBL,

2. DMPK/Physchem data, deposited by AstraZeneca.

Here we were interested in observing in which way additional data could help to improve the model. Separating the

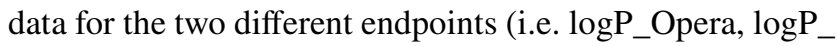
ChEMBL) outperformed aggregating all public data in one endpoint (i.e. $\log P$ _Opera + ChEMBL), but there is no substantial difference between model 5 and the default model (RMSE model $5=0.47$; RMSE model $1=0.45$ ). Expecting that generalizability will improve with more, chemically different data sets, subsequent models were developed with all data used to develop model 5 .

The next two models (6 and 7) were developed with ChEMBL data from AstraZeneca for endpoints that may be correlated with $\log \mathrm{P}$ : plasma protein binding, kinetic solubility@pH7.4, logD7.4, and intrinsic (microsomal and hepatocyte) clearance across species. These endpoints were used as "helper tasks." In model 6 we only added the $\log$ D7.4 data, while in model 7 we added all data for the 4 endpoints. This was done to study the effect of including possibly less related tasks. Both models performed comparably and clearly better than model 5 , proving that adding tasks for related properties is beneficial. Model 6 is somewhat better than model 7 (RMSE model $7=0.44$; RMSE model $6=0.42$ ), indicating that adding less related tasks does not provide any benefit. Subsequent models were developed with all data used to develop model 6 . With model 8 , we tried to improve on model 6 by tuning the hyperparameters (doing a run of hyperopt; refer to methods), and using the best settings. Tuning did not improve the model performance for this data set, however. Further work would be needed to assess whether other settings work better.

\section{Using predictions from other models as molecular descriptors or as "helper tasks"}

Because adding AstraZeneca $\log$ D7.4 data led to increased performance, we decided to study the effect of adding calculated helper tasks. For this, predictions made by $\mathrm{S}+\log \mathrm{P}$ and $\mathrm{S}+\log \mathrm{D} 7.4$ were added. These models were chosen for several reasons:

First, because of S+'s excellent neural network-based $\mathrm{pK}_{\mathrm{a}}$ models, which were developed with over 25,000 datapoints [33], and contribute to the accuracy of their $\log$ D7.4 model. Second, perhaps more importantly, because it could help regularize the model, when those properties are added as tasks (i.e. by learning the relationship between $\log \mathrm{P}$ and $\log \mathrm{D})$. Based on this we hypothesized that adding both $\mathrm{S}+\log \mathrm{P}$ and $\log \mathrm{D} 7.4$ predictions could help improve performance.

Indeed, as shown in Table 1 , adding $\mathrm{S}+\log \mathrm{P}$ and $\mathrm{S}+\log$ D7.4 either as "helper tasks" (model 9) or as molecular descriptors (model 10) improves the performance of the model. One significant practical benefit of adding $\log \mathrm{P}$ and $\log$ 7.4 as helper tasks rather than as descriptors, however, is that the helper tasks are only necessary to optimize the neural network (to make the model more robust and generalizable), but are not used to make actual model predictions. For that reason, slow models used as helper tasks can be tolerated.

Finally, we compared the performance of multitask ensemble model 12 (model 9, but an ensemble of 10) with equivalent singletask ensemble model 11 (default model 1, but an ensemble of 10). The former model performed better, illustrating that the helper tasks and additional data did have a positive impact on the test set performance. The final performance of the model on the test set is shown in Fig. 1.

\section{Performance of the final full model on external sets}

Our primary goal was to develop a model that would be both generally applicable and perform well in the SAMPL7 challenge. As indicated in Methods, we developed two models on the basis of all available data (without having separate training and test sets): multitask ensemble model 12_Full and-for comparison—singletask ensemble model 11_Full. The performance of model 12_Full on the SAMPL7 compounds is shown in Fig. 2. Compared to other SAMPL7 competitors, our final multitask model (12_Full) performs very well: it ranked 2nd out of 17 ranked submissions, and 4 th out of all 36 submissions. To assess the general applicability of our method we further benchmarked model 12_Full by applying it to several external data sets. Results for the SAMPL6 [12], SAMPL7, and Martel et al. [4] data sets are shown in Table 2 . To verify that our observations regarding the benefits of adding extra data and using helper tasks were not limited to one data set, we also applied singletask model 11_Full to these external data sets. In addition, we established a baseline by applying the commercial models (AlogP, XlogP3 and $\mathrm{S}+\log \mathrm{P}$ ).

On both the SAMPL6 challenge and SAMPL7 challenge data sets multitask model 12_Full performed better than all other models we compared it to (singletask model 11_Full, AlogP, X $\log \mathrm{P} 3$ and $\mathrm{S}+\log \mathrm{P}$ ). In fact, for the SAMPL6 compound set, retrospectively analyzed, model 12_Full would have ranked number one, with an RMSE of 0.34, outperforming other methods like cosmotherm_FINE19 (RMSE: 
Fig. 1 Scatter plot of the performance of the final model (Experimental $\log \mathrm{P}$ versus Predicted $\log \mathrm{P}$ ) on the test set. On the top a distribution histogram of the predictions is shown and on the right a distribution histogram of the experimental values. The shaded area (very close to the identity line) represents the $95 \%$ confidence interval for the regression estimate

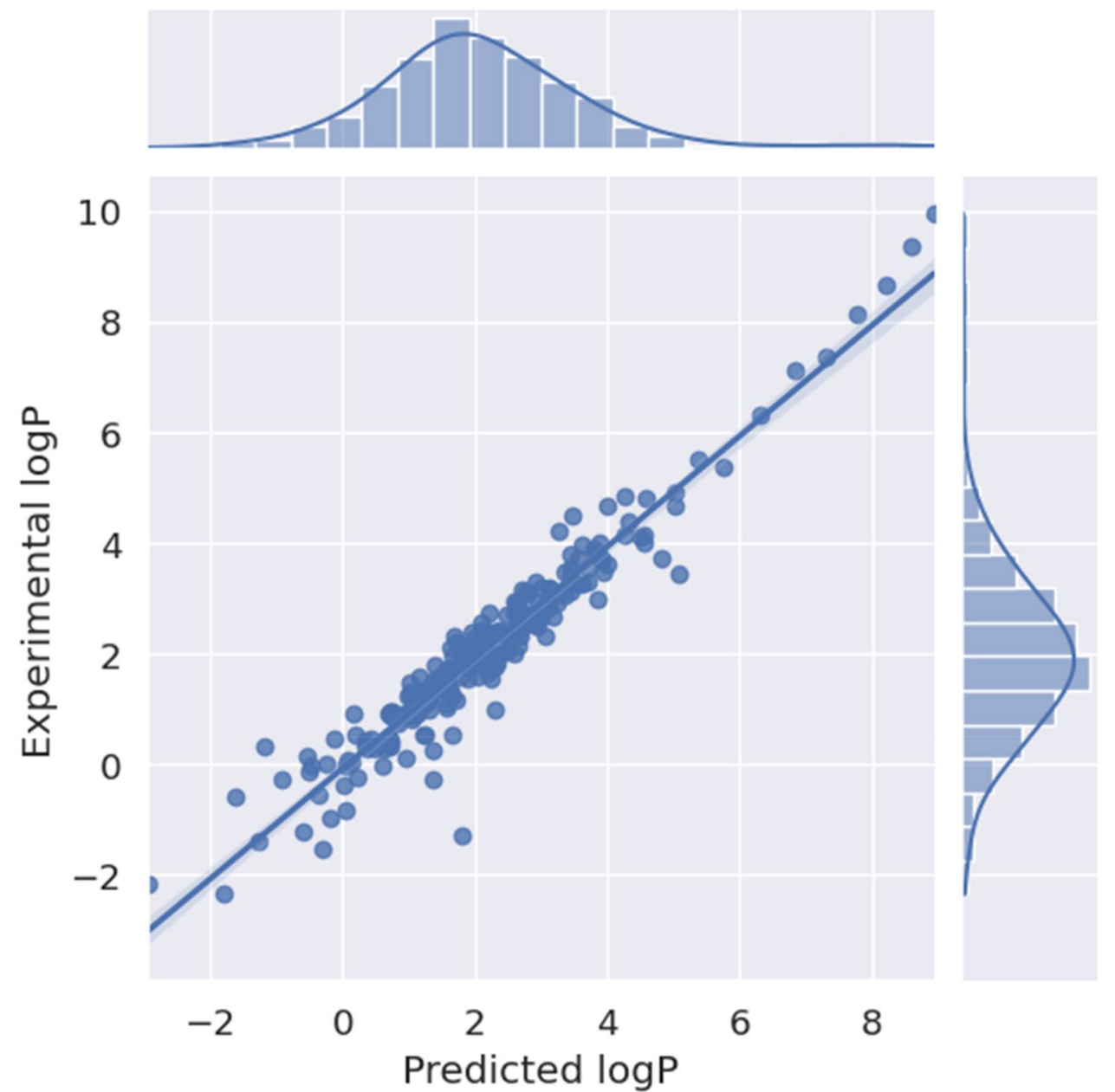

0.38), and the global Xgboost-based QSPR model (RMSE: 0.39) [12].

For the Martel data set model 12_Full ranked second, close in performance to XlogP3. This dataset has been described as a challenging dataset, and in terms of absolute $\mathrm{R}^{2}$ and $\mathrm{RMSE}$ values, none of the five models perform adequately. More work would be needed to understand the poor performance of all models on the Martel data set, but that is beyond the scope of this paper.

In all cases the multitask model (12_Full) outperformed the singletask model (11_Full), although even the latter would have ranked a respectable 11/36 in the SAMPL 7 challenge (considering all submissions).

\section{Analysis of predictions in terms of structures}

To further investigate in which cases model 12_Full performs well, and in which cases it does less well, we analyzed the three best and three worst predictions, respectively, for the SAMPL 7 challenge, and compared model 12_Full to two other high-ranking methods from the SAMPL7 challenge (Table 3). Both overpredicted compounds, SM42 and SM43, contain the same substructure, but the shift between the two was well-predicted (i.e. $\Delta \log \mathrm{P}$ (phenyl $\rightarrow N$-dimethyl is 0.91 experimentally and 0.66 predicted by model 12_Full). This suggests that our model 12_Full overestimates the lipophilicity of the phenyl-isoxazole-sulfonamide moiety. Both SM42 and SM43 were well predicted by TFE MLR (ranked first in the SAMPL 7 challenge), which is a multiple linear regression model trained on a set of 82 druglike molecules (60 molecules containing sulfonamides) [34], indicating that for this particular moiety a more general model like ours does not perform as well as a tailor-made model. COSMO-RS [11, 35] exhibited the same behavior as our model, overpredicting both SM42 and SM43.

Perhaps more puzzling is that model 12_Full, COSMORS, and TFE MLR overpredict SM36, while they all correctly predict SM37. This is a similar transformation as SM42 to SM43 (phenyl $\rightarrow N$-dimethyl). In this case, however, the phenyl group has been experimentally determined to be less lipophilic than the $N$-dimethyl moiety $(\Delta \log \mathrm{P}$ (phenyl $\rightarrow N$-dimethyl) is -0.69 experimentally and 0.69 predicted by model 12_Full). Generally, a phenyl group is more lipophilic than a $N$-dimethyl moiety, but this is not 
Fig. 2 Scatter plot of the performance of the final model (Experimental $\log \mathrm{P}$ versus Predicted $\log$ P) on the SAMPL7 molecules. The compounds discussed in the text and shown in Table 3 are labeled. On the top a distribution histogram of the predictions is shown and on the right a distribution histogram of the experimental values. The shaded area represents the 95\% confidence interval for the regression estimate

Table 2 Overview of the performance of the final multitask ensemble model (12_ Full), used for the challenge, the singletask ensemble model (11_ Full), and several commercial $\log$ P prediction tools on the SAMPL7, SAMPL6 and Martel et al. data sets [4]

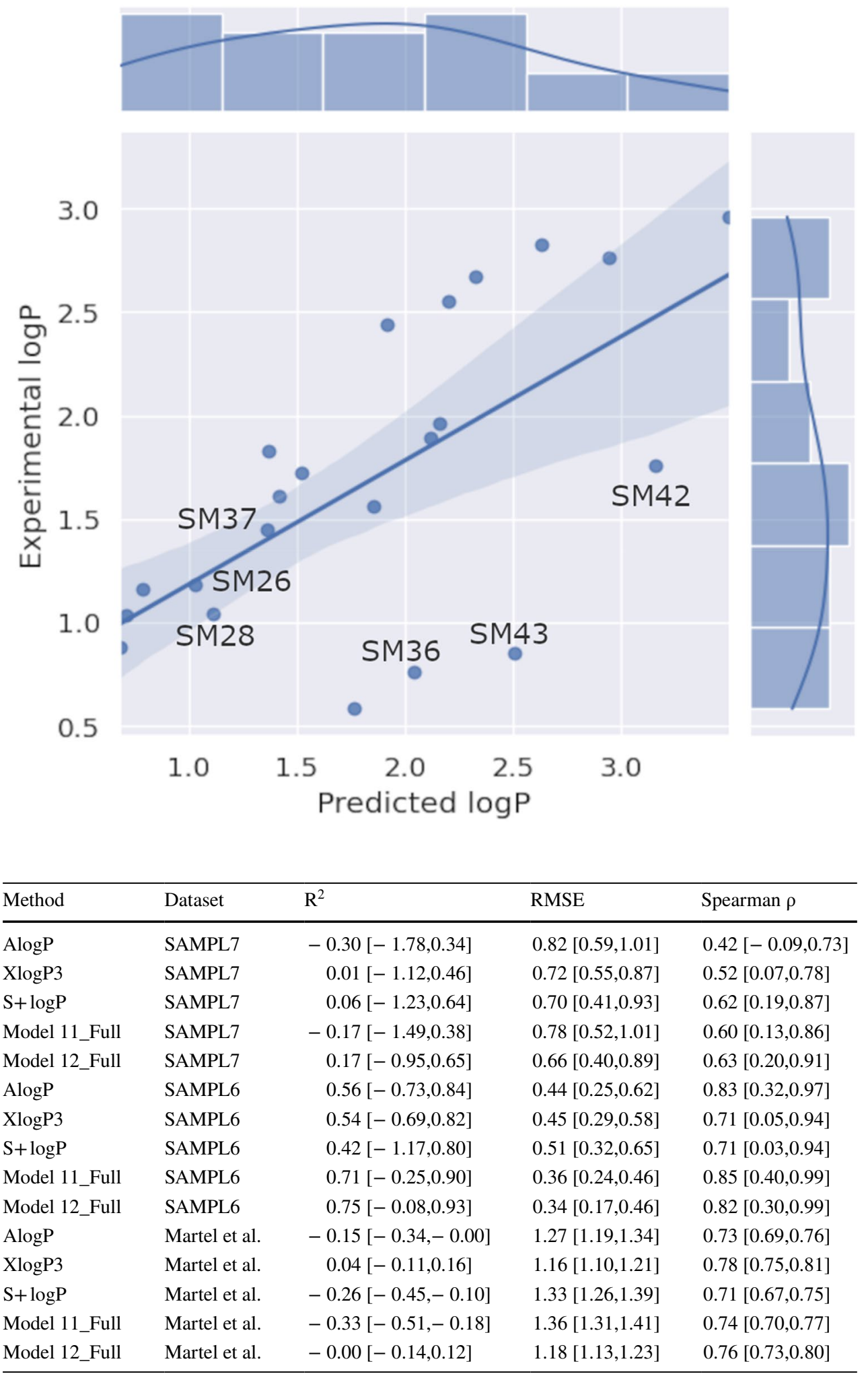

The $95 \%$ confidence interval for the different performance metrics is shown between square brackets. 
Table 3 The top three compounds in terms of largest error (SM43, SM42 and SM36) and lowest error (SM26, SM37 and SM28) for model 12_Full

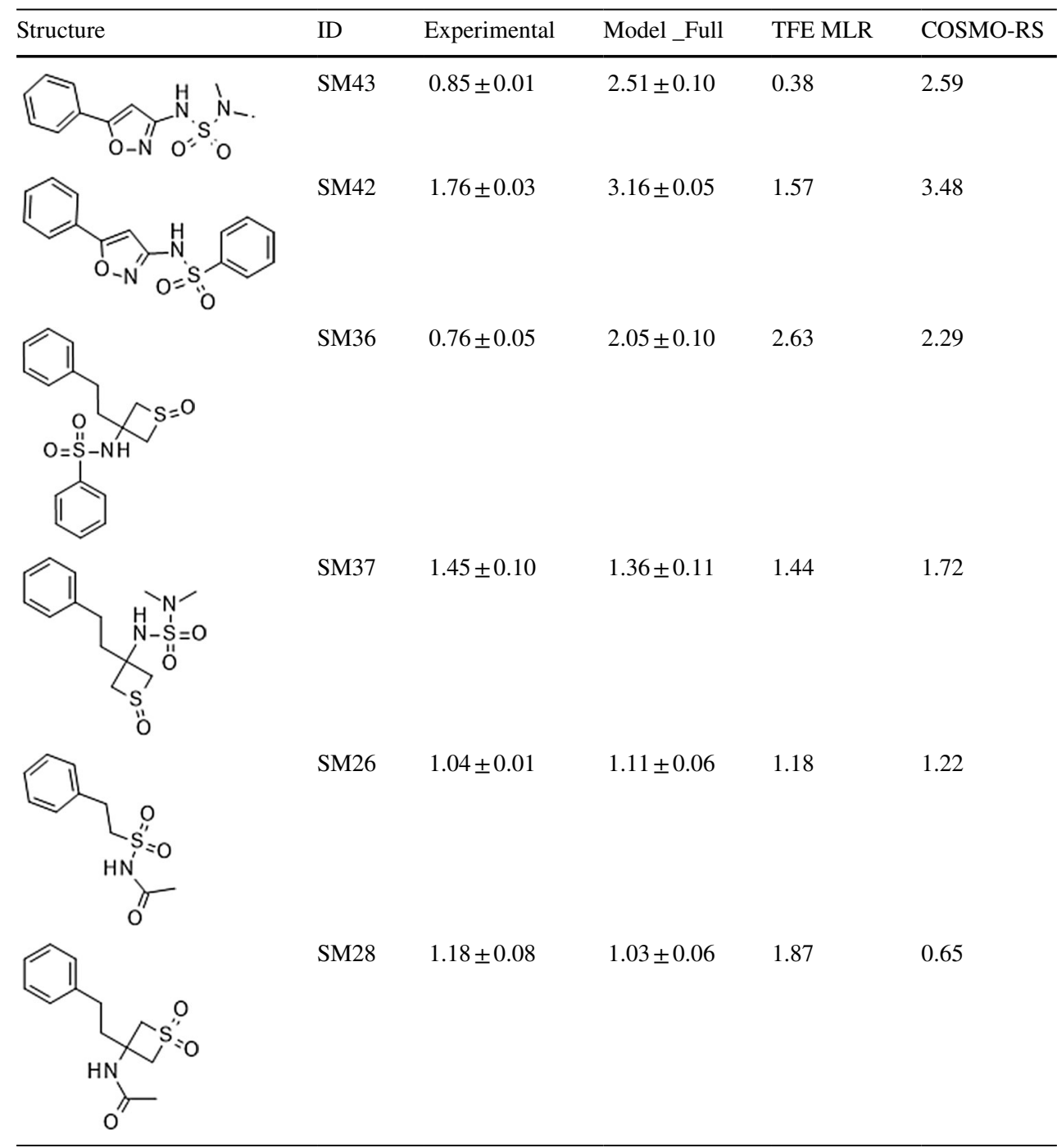

The SEMs for both the experimental data and the predictions by model 12_Full are given behind the \pm sign. Results from two other methods (one statistical, one physical) that participated in the challenge, TFE MLR and COSMO-RS, are shown as a reference $[34,35]$ observed for the latter case. More work would be needed to understand this puzzling outlier.

\section{Discussion and outlook}

This manuscript serves as a walkthrough for the steps that were taken to develop an optimal model, with which to participate in the SAMPL7 challenge. Overall, the model that we developed performs very well, although in most cases only incremental improvements between subsequent models were observed.

Additional combinations can be tested to improve the model performance even more: using additional data (i.e. Martel's) and/or predictions by other models (e.g. XlogP3) may well impact the model performance favorably. Although such a comprehensive investigation is beyond the scope of this paper, it may well be the topic of future work of ours.

In and by itself, the concept of modelling multiple properties as separate tasks within a multitask approach is not novel. What is novel, however, is that here we also consider different datasets for the same property as different tasks (e.g. ChEMBL_logP and Opera_logP). For many other types of assays this kind of data separation should make sense, too: e.g. shake-flask versus chromatographic $\log \mathrm{D}$, thermodynamic versus kinetic solubility, and functional versus binding assays.

Training a $\log \mathrm{P}$ model on the basis of multiple predicted values is not novel per se: this has e.g. been done by JPlogP [36]. However, to the best of our knowledge, the addition of calculated properties as helper tasks to a multitask model is novel, and we expect it to have a wider applicability. Since 
these tasks only need to be calculated once, comprehensive further studies can easily and should be done to investigate whether the models improve if a larger set of (diverse) predictors were added as helper tasks.

An additional area of improvement could be the inclusion of physics-based features/predictions. ML models based on QM-derived features, such as ANI [37], allow for rapid estimation of QM-derived features. For ADME modelling QMderived features have indeed improved model performance. Rather than going the QM-ML route, however, one could use other physics-based predictions that provide accurate $\log P$ estimates as additional tasks [38] in the same vein as we describe in this paper.

Finally, one improvement that is absolutely essential (not only for our models) is the proper estimation of the uncertainty of the predictions. Recently bayesian-based approaches have been described [39], also e.g. complementary to D-MPNN [40]. If uncertainties of the predictions could be accurately estimated this would impact in several ways: for instance to decide which compounds need to be made/tested in drug discovery projects, but also to decide which compounds need to be made/tested in order to improve the model.

\section{Conclusions}

In this manuscript we have discussed the steps that were taken to create an optimal D-MPNN based logP prediction model. This model was constructed for the SAMPL7 challenge, where it scored 2/17 in ranked submissions, and 4/36 in all submissions. Three key improvements over the default D-MPNN model were the result of using: (1) additional data sets for the same and related properties as helper tasks, (2) predicted properties $(\mathrm{S}+\log \mathrm{P} / \log \mathrm{D} 7.4)$ as helper tasks, and (3) an ensemble of models. In a retrospective analysis the model also outperformed other methods when applied to the compounds from the previous SAMPL6 challenge. Performance was second of the methods applied to the Martel data set, but not very good in absolute terms, indicating that further work is warranted. Based on our results, we are convinced that ensembles of multitask models, developed with helper tasks and employing predictions by other models for related properties have great potential application well beyond modelling $\log \mathrm{P}$.

Acknowledgements We thank Miriam Lopez Ramos, Giovanni Tricarico, Thomas Coudrat and Willem Jespers for fruitful discussions.

Author contributions EBL performed the experiments and the analyses. PFWS and EBL defined the scope and strategy of the work, and wrote the manuscript.
Data availability The data used in training the final model, and final models will be shared on github: https://github.com/lenselinkbart/ SAMPL7_paper.

Open Access This article is licensed under a Creative Commons Attribution 4.0 International License, which permits use, sharing, adaptation, distribution and reproduction in any medium or format, as long as you give appropriate credit to the original author(s) and the source, provide a link to the Creative Commons licence, and indicate if changes were made. The images or other third party material in this article are included in the article's Creative Commons licence, unless indicated otherwise in a credit line to the material. If material is not included in the article's Creative Commons licence and your intended use is not permitted by statutory regulation or exceeds the permitted use, you will need to obtain permission directly from the copyright holder. To view a copy of this licence, visit http://creativecommons.org/licenses/by/4.0/.

\section{References}

1. Arnott JA, Planey SL (2012) The influence of lipophilicity in drug discovery and design. Expert Opin Drug Discov 7(10):863-875

2. Tarcsay A, Nyíri K, Keserú GM (2012) Impact of lipophilic efficiency on compound quality. J Med Chem 55(3):1252-1260

3. Ryckmans T, Edwards MP, Horne VA, Correia AM, Owen DR, Thompson LR, Tran I, Tutt MF, Young T (2009) Rapid assessment of a novel series of selective CB2 agonists using parallel synthesis protocols: a lipophilic efficiency (LipE) analysis. Bioorg Med Chem Lett 19(15):4406-4409. https://doi.org/10.1016/j. bmcl.2009.05.062

4. Martel S, Gillerat F, Carosati E, Maiarelli D, Tetko IV, Mannhold R, Carrupt P-A (2013) Large, chemically diverse dataset of $\log$ P measurements for benchmarking studies. Eur J Pharm Sci 48(1-2):21-29

5. Eros D, Kövesdi I, Orfi L, Takács-Novák K, Acsády G, Kéri G (2002) Reliability of $\log P$ predictions based on calculated molecular descriptors: a critical review. Curr Med Chem 9(20):18191829. https://doi.org/10.2174/0929867023369042

6. Yang K, Swanson K, Jin W, Coley C, Eiden P, Gao H, GuzmanPerez A, Hopper T, Kelley B, Mathea M (2019) Analyzing learned molecular representations for property prediction. J Chem Inf Model 59(8):3370-3388

7. Sheridan RP (2013) Time-split cross-validation as a method for estimating the goodness of prospective prediction. J Chem Inf Model 53(4):783-790. https://doi.org/10.1021/ci400084k

8. Mannhold R, Poda GI, Ostermann C, Tetko IV (2009) Calculation of molecular lipophilicity: state-of-the-art and comparison of $\log \mathrm{P}$ methods on more than 96,000 compounds. J Pharm Sci 98(3):861-893. https://doi.org/10.1002/jps.21494

9. Cheng T, Zhao Y, Li X, Lin F, Xu Y, Zhang X, Li Y, Wang R, Lai L (2007) Computation of octanol- water partition coefficients by guiding an additive model with knowledge. J Chem Inf Model 47(6):2140-2148

10. ADMET Predictor v9.5, SimulationsPlus. https://www.simul ations-plus.com/software/admetpredictor/

11. Loschen C, Reinisch J, Klamt A (2020) COSMO-RS based predictions for the SAMPL6 $\log P$ challenge. J Comput Aided Mol Des 34(4):385-392

12. Işık M, Levorse D, Mobley DL et al (2020) Octanol-water partition coefficient measurements for the SAMPL6 blind prediction challenge. J Comput Aided Mol Des 34:405-420

13. Bergazin TD, Tielker N, Zhang Y, Mao J, Gunner MR, Francisco K, Ballatore C, Kast SM, Mobley DL (2021) Evaluation of Log P, PKa, and Log D predictions from the SAMPL7 blind 
challenge. J Comput Aided Mol Des. https://doi.org/10.1007/ s10822-021-00397-3

14. Duvenaud D, Maclaurin D, Aguilera-Iparraguirre J, GómezBombarelli R, Hirzel T, Aspuru-Guzik A, Adams RP (2015) Convolutional networks on graphs for learning molecular fingerprints. arXiv preprint arXiv: 1509.09292

15. McCloskey K, Sigel EA, Kearnes S, Xue L, Tian X, Moccia D, Gikunju D, Bazzaz S, Chan B, Clark MA (2020) Machine learning on DNA-encoded libraries: a new paradigm for hit finding. $\mathrm{J}$ Med Chem 63(16):8857-8866

16. Wu Z, Ramsundar B, Feinberg EN, Gomes J, Geniesse C, Pappu AS, Leswing K, Pande V (2018) MoleculeNet: a benchmark for molecular machine learning. Chem Sci 9(2):513-530. https://doi. org/10.1039/C7SC02664A

17. Stokes JM, Yang K, Swanson K, Jin W, Cubillos-Ruiz A, Donghia NM, MacNair CR, French S, Carfrae LA, Bloom-Ackermann $\mathrm{Z}$ (2020) A deep learning approach to antibiotic discovery. Cell 180(4):688-702

18. Xu Y, Ma J, Liaw A, Sheridan RP, Svetnik V (2017) Demystifying multitask deep neural networks for quantitative structure-activity relationships. J Chem Inf Model 57(10):2490-2504

19. Vamathevan J, Clark D, Czodrowski P, Dunham I, Ferran E, Lee G, Li B, Madabhushi A, Shah P, Spitzer M, Zhao S (2019) Applications of machine learning in drug discovery and development. Nat Rev Drug Discov 18(6):463-477. https://doi.org/10.1038/ s41573-019-0024-5

20. Lenselink EB, Ten Dijke N, Bongers B, Papadatos G, Van Vlijmen HW, Kowalczyk W, IJzerman AP, Van Westen GJ (2017) Beyond the hype: deep neural networks outperform established methods using a ChEMBL bioactivity benchmark set. J Cheminform 9(1):1-14

21. Montanari F, Kuhnke L, Ter Laak A, Clevert D-A (2020) Modeling physico-chemical ADMET endpoints with multitask graph convolutional networks. Molecules 25(1):44

22. Göller AH, Kuhnke L, Montanari F, Bonin A, Schneckener S, Ter Laak A, Wichard J, Lobell M, Hillisch A (2020) Bayer's in silico ADMET platform: a journey of machine learning over the past two decades. Drug Discov Today 25(9):1702-1709. https://doi. org/10.1016/j.drudis.2020.07.001

23. BIOVIA Pipeline Pilot (2021) Release 2016. Dassault Systèmes, San Diego

24. Mansouri K, Grulke CM, Judson RS, Williams AJ (2018) OPERA models for predicting physicochemical properties and environmental fate endpoints. J Cheminform 10(1):1-19

25. Francisco KR, Varricchio C, Paniak TJ, Kozlowski MC, Brancale A, Ballatore C (2021) Structure property relationships of $\mathrm{N}$-acylsulfonamides and related bioisosteres. Eur J Med Chem 218:113399. https://doi.org/10.1016/j.ejmech.2021.113399

26. Rogers D, Hahn M (2010) Extended-connectivity fingerprints. J Chem Inf Model 50(5):742-754

27. Gaulton A, Bellis LJ, Bento AP, Chambers J, Davies M, Hersey A, Light Y, McGlinchey S, Michalovich D, Al-Lazikani B (2012) ChEMBL: a large-scale bioactivity database for drug discovery. Nucleic Acids Res 40(D1):D1100-D1107
28. Landrum G, Tosco P, Kelley B, Sriniker, Gedeck, Schneider N, Vianello R, Ric, Dalke A, Cole B, Savelyev A, Swain M, Turk S, Dan N, Vaucher A, Kawashima E, Wójcikowski M, Probst D, Godin G, Cosgrove D, Pahl A, JP, Berenger F, strets123, Varjo JL, O'Boyle N, Fuller P, Jensen JH, Sforna G, Gavid D (2020) Rdkit/ Rdkit: 2020_03_1 (Q1 2020) Release. Zenodo. https://doi.org/10. 5281/zenodo.3732262

29. Bergstra J, Komer B, Eliasmith C, Yamins D, Cox DD (2015) Hyperopt: a python library for model selection and hyperparameter optimization. Comput Sci Discov 8(1):014008

30. Pedregosa F, Varoquaux G, Gramfort A, Michel V, Thirion B, Grisel O, Blondel M, Prettenhofer P, Weiss R, Dubourg V (2011) Scikit-learn: machine learning in python. J Mach Learn Res 12:2825-2830

31. Raschka S (2018) MLxtend: providing machine learning and data science utilities and extensions to python's scientific computing stack. J Open Source Softw 3(24):638

32. Ghose AK, Viswanadhan VN, Wendoloski JJ (1998) Prediction of hydrophobic (lipophilic) properties of small organic molecules using fragmental methods: an analysis of ALOGP and CLOGP methods. J Phys Chem A 102(21):3762-3772

33. Fraczkiewicz R, Lobell M, Göller AH, Krenz U, Schoenneis R, Clark RD, Hillisch A (2015) Best of both worlds: combining pharma data and state of the art modeling technology to improve in silico pKa prediction. J Chem Inf Model 55(2):389-397

34. Lopez Perez K, Pinheiro S, Zamora W (2021) Multiple linear regression models for predicting the $\mathrm{N}$-octanol/water partition coefficients in the SAMPL7 blind challenge. J Comput Aided Mol Des

35. Warnau J, Wichmann K, Reinisch J (2021) COSMO-RS predictions of $\log \mathrm{P}$ in the SAMPL7 blind challenge. J Comput Aided Mol Des

36. Plante J, Werner S (2018) JPlogP: an improved $\log \mathrm{P}$ predictor trained using predicted data. J Cheminform 10(1):61. https://doi. org/10.1186/s13321-018-0316-5

37. Smith JS, Zubatyuk R, Nebgen B, Lubbers N, Barros K, Roitberg AE, Isayev O, Tretiak S (2020) The ANI-1ccx and ANI-1x data sets, coupled-cluster and density functional theory properties for molecules. Sci Data 7(1):1-10

38. Göller AH (2019) The art of atom descriptor design. Drug Discov Today Technol 32-33:37-43. https://doi.org/10.1016/j.ddtec. 2020.06.004

39. Zhang Y, Lee AA (2019) Bayesian semi-supervised learning for uncertainty-calibrated prediction of molecular properties and active learning. Chem Sci 10(35):8154-8163. https://doi.org/10. 1039/C9SC00616H

40. Lamb G, Paige B (2020) Bayesian graph neural networks for molecular property prediction. arXiv preprint arXiv:2012.02089

Publisher's Note Springer Nature remains neutral with regard to jurisdictional claims in published maps and institutional affiliations. 